\title{
Mesenchymal Stem Cell-Educated Macrophages Ameliorate LPS-Induced Systemic Response
}

\author{
Yaoqin Hu, ${ }^{1}$ Chaojin Qin, ${ }^{1}$ Guoping Zheng, ${ }^{2}$ Dengming Lai, ${ }^{1}$ \\ Huikang Tao, ${ }^{1}$ Yan Zhang, ${ }^{1}$ Guanguan Qiu, ${ }^{2}$ Menghua Ge, ${ }^{2}$ Lanfang Huang, \\ Lina Chen, ${ }^{2}$ Baoli Cheng, ${ }^{3}$ Qiang Shu, ${ }^{1}$ and Jianguo $\mathrm{Xu}^{2,3}$ \\ ${ }^{1}$ The Children's Hospital of Zhejiang University School of Medicine, Hangzhou, Zhejiang 310052, China \\ ${ }^{2}$ Shaoxing Second Hospital, Shaoxing, Zhejiang 312000, China \\ ${ }^{3}$ The First Affiliated Hospital of Zhejiang University School of Medicine, Hangzhou, Zhejiang 310003, China \\ Correspondence should be addressed to Qiang Shu; shuqiang@zju.edu.cn and Jianguo Xu; jxu5@yahoo.com
}

Received 1 January 2016; Revised 23 May 2016; Accepted 6 June 2016

Academic Editor: Anshu Agrawal

Copyright (C) 2016 Yaoqin Hu et al. This is an open access article distributed under the Creative Commons Attribution License, which permits unrestricted use, distribution, and reproduction in any medium, provided the original work is properly cited.

Both bone marrow and adipose-derived mesenchymal stem cells (ASCs) have immunomodulatory effects. The goal of this study was to determine whether ASCs-educated macrophages could directly ameliorate LPS-induced systemic response in a mouse model. Mouse peritoneal macrophages were cocultured with ASCs in a Transwell system for 2 days to educate macrophages. Mice were divided into 5 groups: control, LPS, LPS + ASCs, LPS + untreated macrophages, and LPS + educated macrophages. Educated macrophages decreased lung inflammation, weight loss, pulmonary edema, and inflammatory cytokine response. In vitro, ASCs increased expression of M2 macrophages independent of direct cell-to-cell contact when macrophages were treated with LPS or serum from patients with acute respiratory distress syndrome (ARDS). When macrophages were cultured with serum from ARDS patients who were treated with ASCs or placebo in our previous clinical trial, there was no difference in M2 macrophage levels before and after ASCs treatment indicating a suboptimal response to the treatment protocol. ASCs also reduced the levels of LPSinduced proinflammatory cytokines in vitro which were mimicked by IL-10 and blocked by antibodies for IL-10 and IL-10 receptor supporting the notion that educated macrophages exert their anti-inflammatory effects via IL-10-dependent mechanisms.

\section{Introduction}

Many studies, including publications from our group [1, 2], have demonstrated compelling evidence of the benefits of mesenchymal stem cells (MSCs) from both bone marrow (BM) [3-5] and adipose tissue (adipose-derived mesenchymal stem cells, ASCs) [6-8] in animal models for lung injury and systemic inflammation. The benefit of MSCs appears to derive from the release of paracrine soluble factors such as keratinocyte growth factor [9], angiopoietin-1 [3], and prostaglandin $\mathrm{E}_{2}$ [10]. These factors can reduce inflammation, stabilize the injured alveolar epithelium and lung endothelium, increase the absorption of pulmonary edema fluid, and possess antimicrobial activity [11]. BM-MSCs have also been reported to attenuate sepsis via regulating host macrophages [10]. Recently, BM-MSCs were documented to shuttle mitochondria and microRNAs to macrophages, enhance macrophage bioenergetics, and inhibit macrophage Toll-like receptor (TLR) signaling [12].

Alveolar macrophages are tissue-resident or recruited cells. They form the first line of defense against airborne particles and microbes and use a variety of pattern recognition and scavenger receptors to sense and phagocytize pathogens. Upon activation, they release early response cytokines such as interferon- $\gamma$ (IFN- $\gamma$ ), tumor necrosis factor- $\alpha$ (TNF- $\alpha$ ), and interleukin-1 $\beta$ (IL-1 $\beta$ ) in an interferon regulatory factor (IRF) or nuclear factor-kappa B- (NF- $\kappa \mathrm{B}-$ ) dependent way. These cytokines stimulate neighboring alveolar epithelial cells and macrophages in an autocrine and paracrine manner to produce a variety of chemokines which in turn mediate the recruitment of neutrophils. Later on, macrophages and lymphocytes are attracted to the site of infection and facilitate 
the clearance of pathogens [13]. Macrophages are divided into two general categories: classical activation M1 and alternative activation M2. Classical M1 activation is driven by IFN- $\gamma$ and TLR ligands and is an aggressive state in which macrophages attack and engulf bacteria or bacterially infected cells. M1 macrophages secrete cytokines that promote inflammation, such as IFN- $\gamma$, IL- 6 , and TNF- $\alpha$. Alternative M2 activation describes the behavior of macrophages reacting to parasitic infection, but it is also associated with wound healing and lung fibrosis, and results in the production of antiinflammatory cytokines such as IL-10. The M1 and M2 classifications are useful in describing proinflammatory (M1) and anti-inflammatory (M2) states [14].

The concept for "MSCs-educated macrophages" was first proposed by Kim et al. [15, 16]. They first cultured human monocytes from peripheral blood without any added cytokines to generate macrophages and then cocultured macrophages for 3 more days with culture-expanded BM-MSCs. Macrophages cocultured with MSCs consistently showed high-level expression of CD206, a marker of alternatively activated macrophages. Furthermore, these macrophages expressed high levels of IL-10 and low levels of IL-12, as determined by intracellular staining, typical of alternatively activated macrophages [15]. Anderson et al. reported that systemic infusion of macrophages pretreated with ASCsconditioned medium inhibited colitis and polymicrobial sepsis induced by cecal ligation and puncture [17]. In another study, educated macrophages ameliorated rhabdomyolysisinduced acute kidney injury [18]. The present study was designed to test the hypothesis that educated macrophages mimic the effects of ASCs in reducing systemic response induced by LPS.

\section{Materials and Methods}

2.1. Human Samples. Human protocol was approved by the Research Ethics Committee at Shaoxing Second Hospital. Pooled ARDS serum samples were obtained from 3 patients treated at the ICU unit of Shaoxing Second Hospital. All subjects developed ARDS secondary to bacterial pneumonia. Subjects were mechanically ventilated for 2-4 days. The severity of ARDS was classified as moderate to severe based on the Berlin definition [19]. Serum samples from ARDS patients treated with placebo and ASCs were obtained from a clinical trial conducted by our group [20].

2.2. Mice. C57BL/6 mice (8-12 weeks old; Shanghai Laboratory Animal Center, Shanghai, China) were used for the study. All mice were housed in the Zhejiang University Laboratory Animal Center. Animal experiment protocols were approved by the review committee from Zhejiang University School of Medicine and were in compliance with institutional guidelines.

2.3. Cell Culture. For macrophages, C57BL/6 mice were injected intraperitoneally with $2 \mathrm{~mL}$ of $3 \%$ thioglycollate. After 3 days, the peritoneal macrophages were collected by lavage, characterized, and counted using a Beckman Coulter counter. Macrophages were maintained in medium containing Dulbecco's Modified Eagle's Medium- (DMEM-) low glucose supplemented with $1 \%$ penicillin and streptomycin and $8 \%$ fetal bovine serum (FBS) (Life Technologies, Grand Island, NY) plus EGF and FGF (R\&D Systems, Minneapolis, $\mathrm{MN}$ ) at $37^{\circ} \mathrm{C}$ in a humidified incubator containing $5 \% \mathrm{CO}_{2}$. Normal human ASCs were purchased from ATCC (Cat. number PCS-500-011, passage 2, Manassas, VA). Cells were cultured in the same culture medium above at a density of 4000 cells $/ \mathrm{cm}^{2}$. Cultures were maintained at $37^{\circ} \mathrm{C}$ in a humidified atmosphere containing $5 \% \mathrm{CO}_{2}$ in $150 \mathrm{~mm}$ dishes (Life Technologies, Grand Island, NY). When the cultures reached near confluence $(>80 \%)$, the cells were detached by treatment with trypsin/EDTA and replated at a density of 4000 cells $/ \mathrm{cm}^{2}$. The ASCs were passaged up to a maximum of four times. These cells have been characterized in our previous publication [20]. Methods for culture of educated macrophages were modified from the techniques published by Kim and Hematti [15]. Macrophages $\left(2 \times 10^{6}\right.$ cells/well $)$ were placed at the bottom of 6 well plates. Then, ASCs $\left(5 \times 10^{5}\right.$ cells/well, a ratio of $4: 1)$ were added on the top of Transwell $(0.4 \mu \mathrm{m}$ pore size; Costar; Corning) system. After 48 hours of coculture, macrophages were harvested and designated as "educated macrophages" and used for in vivo experiment.

2.4. LPS-Induced Systemic Response. LPS from Escherichia coli O111:B4 (Sigma, St. Louis, MO) was dissolved with phosphate-buffered saline (PBS) by $1 \mathrm{mg} / \mathrm{mL}$. Mice were randomly assigned to one of five experimental groups: (1) PBS control; (2) LPS; (3) LPS + ASCs; (4) LPS + untreated macrophages; or (5) LPS + educated macrophages. Mice were inoculated intraperitoneally with $5 \mathrm{mg} / \mathrm{kg}$ LPS or PBS. Before infusion, ASCs, educated macrophages, and untreated macrophages were washed with warm PBS and resuspended at a concentration of $1 \times 10^{6}$ cells per $0.2 \mathrm{~mL}$ of PBS. Immediately after PBS or LPS treatment, $1 \times 10^{6}$ cells (ASCs, educated macrophages, or untreated macrophages) or PBS $(0.2 \mathrm{~mL})$ were injected via the tail veins of the mice. Animals were weighed and sacrificed before and at 6,24, and $48 \mathrm{~h}$ after LPS or PBS treatment. Lungs were harvested for histological analysis and determination of the wet-dry ratio. Blood and bronchial alveolar lavage (BAL) samples were collected from animals for cytokine analysis.

2.5. Wet-Dry Analysis. Lungs were removed from mice, placed into microcentrifuge tubes, and weighed. Lungs were then desiccated at $85^{\circ} \mathrm{C}$ oven for 48 hours for dry weight measurement. The wet-dry ratio was determined by dividing the weight before and after desiccating.

2.6. Histopathology. To harvest the lungs, the tracheas were cannulated and the lungs fixed by inflation with $4 \%$ paraformaldehyde. Following overnight fixation, tissue was embedded in paraffin and sectioned at $5 \mu \mathrm{m}$ thickness. Hematoxylin and eosin (H\&E) staining was performed to determine morphology and inflammatory infiltrate. For measurement of the number of neutrophils in the lungs, modifications were made from the technique published by 
Everhart et al. [21]. Briefly, numbers of neutrophils were counted at 10 randomly selected high-power magnification fields $(\times 400)$ in three histological sections per mouse from a total of four mice.

2.7. Detection of Cytokines. BAL samples were collected using a standard protocol. Mouse blood samples were collected and allowed to clot at room temperature for 30-90 min, followed by centrifugation at $400 \mathrm{~g}$ for $6 \mathrm{~min}$. The serum and supernatants from BAL samples were then aliquoted and stored at $-80^{\circ} \mathrm{C}$. All the samples were analyzed for IL- $1 \beta$, IL4, IL-6, IL-10, IL-13, IL-17A, IFN- $\gamma$, TNF- $\alpha$, and MIP- $1 \alpha$ via a ProcartaPlex ${ }^{\mathrm{TM}}$ Multiplex Immunoassay (eBioscience, San Diego, CA) according to the manufacturer's protocol. The assay plate was read on the Luminex 200 System (Luminex, Austin, TX). Standards and samples were run in duplicate and analyzed using the ProcartaPlex Analyst Software 1.0 .

2.8. Coculture Experiments. Mouse peritoneal macrophages $\left(2 \times 10^{6}\right)$ were cultured alone or cocultured with ASCs $(5$ $\left.\times 10^{5}\right)$ in either a standard 6 -well or in a Transwell $(0.4 \mu \mathrm{m}$ pore size; Costar; Corning) system for 48 hours. Macrophages were then stimulated with or without LPS $(10 \mathrm{ng} / \mathrm{mL})$ for 24 hours. The cell culture supernatants were harvested and stored at $-80^{\circ} \mathrm{C}$ for future cytokine analysis. Macrophages were treated with trypsin and harvested for phenotype analysis. For the ARDS serum experiment, macrophages were incubated for $24 \mathrm{~h}$ with fresh culture medium plus $8 \%$ FBS (M group), normal human serum, or serum from ARDS patients. Then, macrophages were collected for phenotype analysis. For the IL-10 blockage experiment, macrophages were indirectly cocultured with ASCs via Transwell system in the presence or absence of IL-10 antibody $(10 \mathrm{ng} / \mathrm{mL})$ (mouse monoclonal antibody, R\&D Systems, MAB417), IL10 receptor antibody $(10 \mathrm{ng} / \mathrm{mL})$ (mouse polyclonal antibody, R\&D Systems, AF-474-NA), or mouse IgG1 control $(10 \mathrm{ng} / \mathrm{mL})(\mathrm{R} \& \mathrm{D}$ Systems). After $48 \mathrm{~h}$, cells were replenished with fresh medium and treated with LPS $(10 \mu \mathrm{g} / \mathrm{mL})$, and treatment was continued with IL-10 antibody, IL-10 receptor antibody, or IgG1 control for $24 \mathrm{~h}$. Culture supernatants were harvested for cytokine assay. For the IL-10 treatment experiment, macrophages $\left(2 \times 10^{6}\right.$ cells/well $)$ were pretreated with or without IL-10 (1 ng/mL and $10 \mathrm{ng} / \mathrm{mL})$ for 6 hours and then incubated with LPS $(10 \mathrm{ng} / \mathrm{mL})$ for another 24 hours. Culture supernatants and macrophages were harvested for mechanistic analysis.

2.9. Flow Cytometry. For determination of M2 macrophage expression, cells were labeled with FITC anti-mouse CD68 (BioLegend, San Diego, CA) and APC anti-mouse CD206 (BioLegend). After incubation with antibodies at $4^{\circ} \mathrm{C}$ for $30 \mathrm{~min}$, cells were washed with PBS supplemented with $2 \%$ BSA, $4 \mathrm{mM}$ EDTA, and $0.01 \% \mathrm{NaN} 3$ and fixed in $4 \%$ paraformaldehyde. Data were collected on a FACSCalibur (BD Biosciences) and analyzed using FlowJo software.
2.10. Statistical Methods. Data are expressed as mean \pm standard error of the mean (SEM). Statistical analysis was carried out using the GraphPad Prism software. Comparisons were analyzed by one-way ANOVA with a Bonferroni post hoc test or Student's $t$-test. Values were considered significant if $p<0.05$.

\section{Results}

3.1. Educated Macrophages Mimic the Effect of ASCs in LPS-Induced Lung Inflammation. To determine whether administration of educated macrophages alters LPS-induced lung inflammation, mice were randomized to the following 5 groups: control, LPS, LPS + ASCs, LPS + untreated macrophages (M), and LPS + educated macrophages (EM). At the 24 h time point, histological sections stained with $\mathrm{H} \& \mathrm{E}$ are shown in Figure 1(a). Administration of LPS revealed evidences of lung inflammation including marked inflammatory infiltrates, alveolar septal thickening, and interstitial edema. Infusion of ASCs or educated macrophages reduced airspace inflammation. Infusion of untreated macrophages did not inhibit the effect of LPS in the lung. To quantify the effect of educated macrophages on lung inflammation, a blinded histopathological examination of lung sections was carried out and the neutrophils in histological sections were counted at the $24 \mathrm{~h}$ time point (Figure 1(b)). Significant decreases in the number of neutrophils in animals treated with ASCs or educated macrophages were revealed as compared with LPS alone.

ASCs have been shown to alleviate the weight loss of LPSinjured mice [22]. Educated macrophages had a similar effect to that of ASCs in reducing weight loss at $24 \mathrm{~h}$ time point (Figure 2(a), $p<0.05)$. Pulmonary edema is a hallmark of lung edema and inflammation. Figure 2(b) summarized the time course of wet-dry weight ratios of lungs from all 5 groups. In mice treated with LPS alone, edema reached a peak at $24 \mathrm{~h}$ and was largely resolved by $48 \mathrm{~h}$, which is similar to the animals that received LPS + M. No edema developed in the lungs of animals received LPS + ASCs and LPS + EM. These findings demonstrate that educated macrophages reduce LPS-induced lung inflammation.

\subsection{Educated Macrophages Alleviate the LPS-Induced Systemic} Inflammatory Response. To evaluate the anti-inflammatory actions by ASCs and educated macrophages, levels of proinflammatory and anti-inflammatory cytokines were measured in serum and BAL collected from the above animals. Six hours after LPS administration, treatment with ASCs and educated macrophages significantly attenuated the levels of LPS-induced proinflammatory cytokine IFN- $\gamma$ and IL-6 while increasing the level of anti-inflammatory cytokine IL-10 in the serum (Figure 3(a)). ASCs and educated macrophages also significantly decreased IL-6 level and elevated levels of IL-4, IL-10, and IL-13 in the BAL (Figure 3(b)). This response peaked at $6 \mathrm{~h}$ after LPS administration and largely subsided by $48 \mathrm{~h}$. The effect of ASCs observed in the present study correlates well with the literature $[8,22]$. These results 


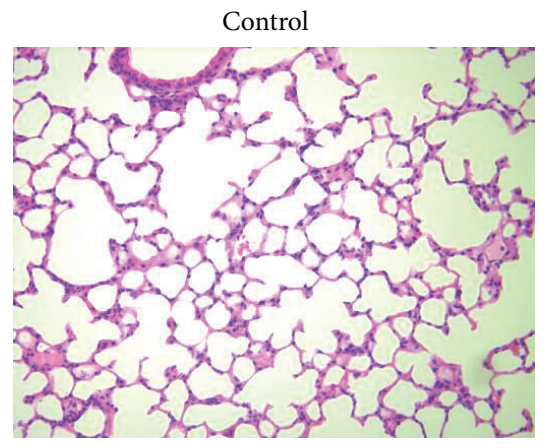

LPS

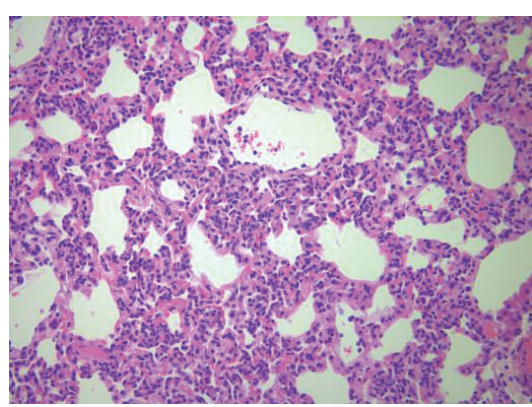

LPS + ASCs

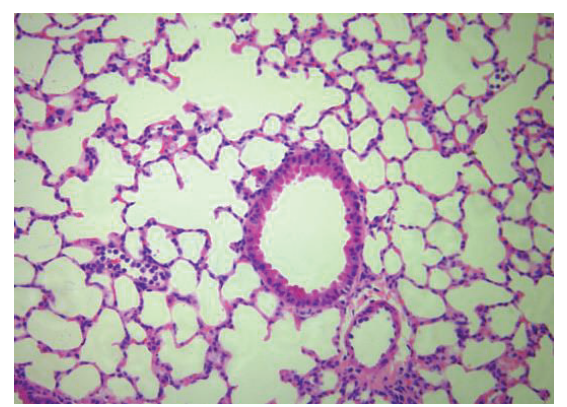

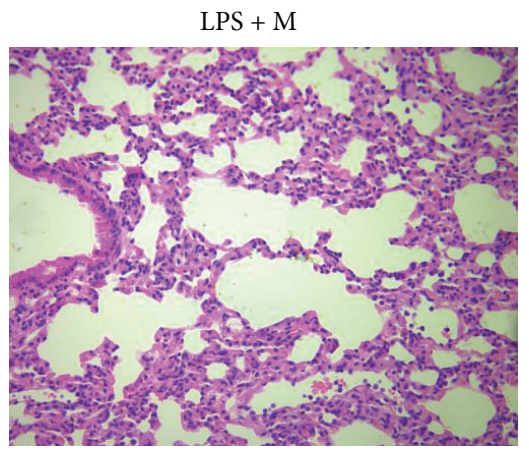

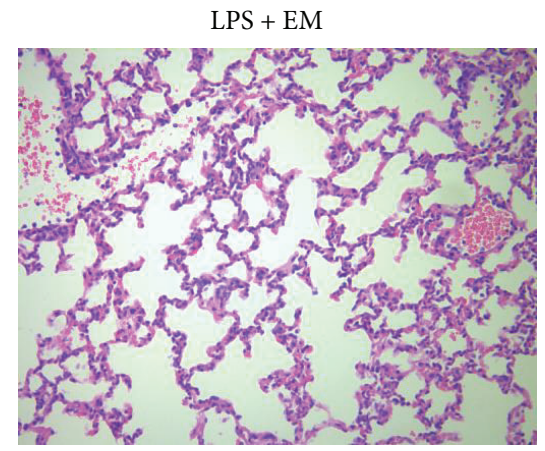

(a)

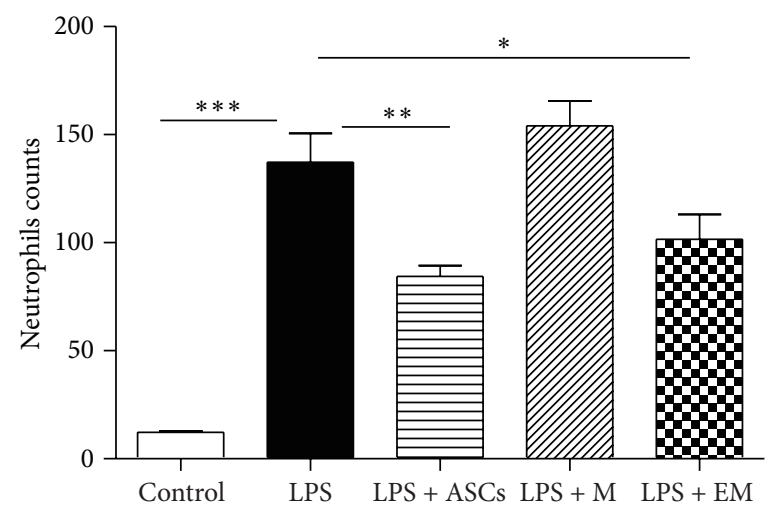

(b)

FIGURE 1: Educated macrophages (EM) decrease LPS-induced lung inflammation. Mice were allocated and received the following treatment: control, LPS, LPS + ASCs, LPS + macrophages (M), and LPS + EM. Animals were sacrificed before and at 6, 24, and 48 h after LPS or PBS treatment. (a) Lungs were fixed in paraformaldehyde. Lung sections were stained with H\&E and visualized at $\times 200$ magnification. (b) Quantification of neutrophils per high-power field on lung sections stained with H\&E. All data are expressed as mean \pm SEM. ${ }^{*} p<0.05$; ${ }^{* *} p<0.01 ;$ and $^{* * *} p<0.001$.

demonstrate that educated macrophages reduce LPS-induced systemic cytokine response.

3.3. ASCs Elevate Expression of M2 Macrophages Independent of Direct Cell-to-Cell Contact. To determine the mechanisms of ASCs in alleviating systemic inflammatory response, we studied cell surface expression of $\mathrm{CD}^{+} 8^{+} \mathrm{CD} 206^{+}$, a well known marker for M2 macrophages [23]. Macrophages were cultured alone, cocultured for $48 \mathrm{~h}$ with ASCs via Transwell or direct coculture and incubated with or without LPS $(10 \mathrm{ng} / \mathrm{mL})$ for an additional $24 \mathrm{~h}$. LPS treatment significantly decreased the levels of $\mathrm{CD}^{+} 8^{+} \mathrm{CD} 206^{+}$cells when macrophages were cultured alone (Figures 4(a) and 4(b)). Both Transwell and direct culture with ASCs reversed the effect of LPS on $\mathrm{CD} 68^{+} \mathrm{CD} 206^{+}$cells. The data suggest that paracrine factors are involved in the increased M2 expression.

3.4. ASCs Increase M2 Expression in Macrophages Treated with ARDS Serum. To investigate the mechanism of ASCs for the potential treatment of ARDS, macrophages were cultured with medium containing $8 \%$ fetal bovine serum or pooled ARDS serum collected at the peak of clinical symptoms. Similar to LPS, ARDS serum significantly reduced 


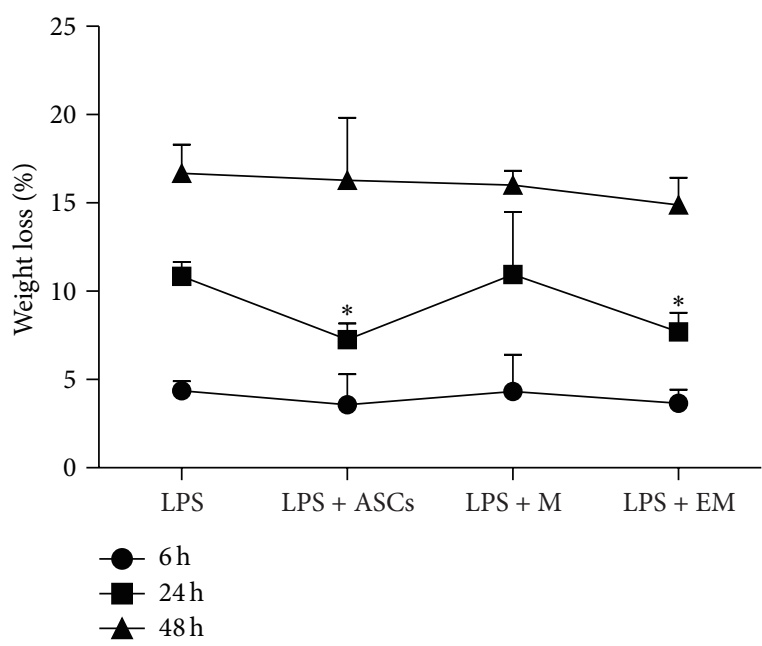

(a)

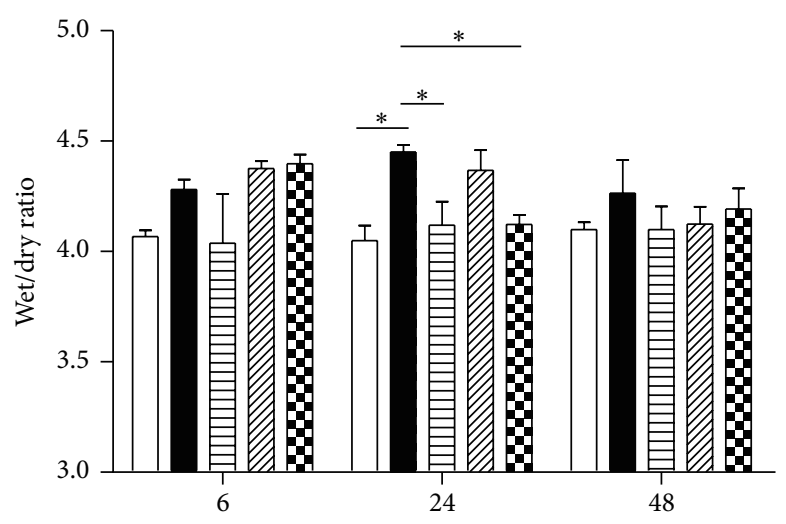

(h)

WIIT LPS $+\mathrm{M}$

00 LPS + EM

(b)

FIGURE 2: Infusion of educated macrophages (EM) reduces weight loss and pulmonary edema in mice treated with LPS. Animals were sacrificed before and at 6, 24, and $48 \mathrm{~h}$ after LPS or PBS treatment. (a) Percentage of weight loss was determined by the lost weight divided by the weight before LPS treatment. (b) Pulmonary edema was measured as the wet-dry ratio. $\mathrm{M}=$ macrophages. All data are expressed as mean \pm SEM; $n=4$ per group. ${ }^{*} p<0.05$.

the expression of $\mathrm{CD} 8^{+} \mathrm{CD} 206^{+}(\mathrm{M} 2)$. Both direct coculture and Transwell of ASCs with macrophages restored the expression of M2 macrophages (Figure 5(a)). In a clinical trial for ARDS conducted by our group, SP-D levels, a marker for ARDS, were significantly reduced after ASCs treatment although no clinical beneficial effect was noticed [20]. The serum samples from patients treated with placebo and ASCs in the trial were cultured with macrophages and examined for M2 expression. There was no significant difference in M2 expression before and after treatment in both placebo and ASCs groups (Figure 5(b)).

3.5. Alterations in the Cytokine Profile Produced by Educated Macrophages Are Mimicked by IL-10 Treatment. To further confirm that ASCs promote the anti-inflammatory M2, the secretion of cytokines in cultured macrophages was examined. Macrophages were cultured for 2 days alone, via direct coculture with ASCs or Transwell. Then, cells were treated with or without LPS $(10 \mathrm{ng} / \mathrm{mL})$ for an additional $24 \mathrm{~h}$ and cytokine levels in the supernatants were determined. The levels of proinflammatory IL-6 and TNF$\alpha$ were significantly elevated following LPS treatment and were significantly decreased by direct coculture or Transwell with ASCs (Figure 6(a)). In contrast, the levels of antiinflammatory IL-10 were significantly augmented with both ways of coculture (Figure 6(a)). Similar to the previous findings in the expression of M2 macrophages (Figure 4), there was no statistical difference in cytokine levels (IL-6, TNF- $\alpha$, and IL-10) between direct coculture and Transwell.

IL-10 has been shown to mediate the response of mesenchymal stem cells on macrophages in sepsis [10]. To determine whether IL-10 mediates the alteration of inflammatory cytokines of ASCs, macrophages were cocultured with ASCs via Transwell system in the presence of neutralizing IL-10 antibody $(10 \mathrm{ng} / \mathrm{mL})$ or $\mathrm{IL}-10$ receptor antibody $(10 \mathrm{ng} / \mathrm{mL})$. Cytokine levels in the culture supernatants were analyzed after treatment with LPS for $24 \mathrm{~h}$. Both antibodies for IL-10 and IL-10 receptor significantly impaired the ability of ASCs to inhibit the production of proinflammatory cytokines IL-6 and TNF- $\alpha$ by macrophages (Figure 6(b)).

To further confirm the roles of IL-10 in the educated macrophages, macrophages were cultured with or without IL$10(1 \mathrm{ng} / \mathrm{mL}$ and $10 \mathrm{ng} / \mathrm{mL})$ for 24 hours. M2 expression and cytokine levels in the culture supernatants were determined. Unsurprisingly, IL-10 reduced proinflammatory cytokine IL6 and TNF- $\alpha$ expression (Figure 7(a)). IL-10 also induced M2 phenotype (Figure 7(b)). These findings suggest that ASCs regulate cytokine expression and phenotype in macrophages via an IL-10-dependent mechanism.

\section{Discussion}

In the present study, we tested a new cell-based therapeutic strategy for systemic inflammation using macrophages educated via coculture with ASCs. Our results revealed that educated macrophages directly ameliorated lung inflammation and reduced weight loss as well as pulmonary edema in mice treated with LPS. Educated macrophages also decreased the proinflammatory cytokine levels and increased the antiinflammatory cytokine levels in serum and BAL of the animals. In vitro, ASCs elevated expression of M2 macrophages independent of direct cell-to-cell contact when macrophages were treated with LPS or ARDS serum. The polarization of M2 macrophages in educated macrophages is characterized by a reduced ability to produce proinflammatory cytokines IL- 6 and TNF- $\alpha$ and an increase in IL-10 level. In addition, the 


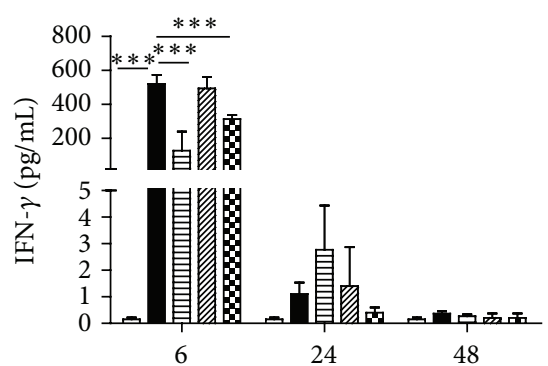

(h)

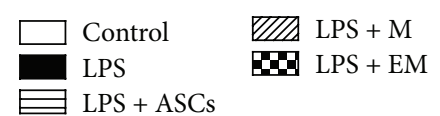

(A)

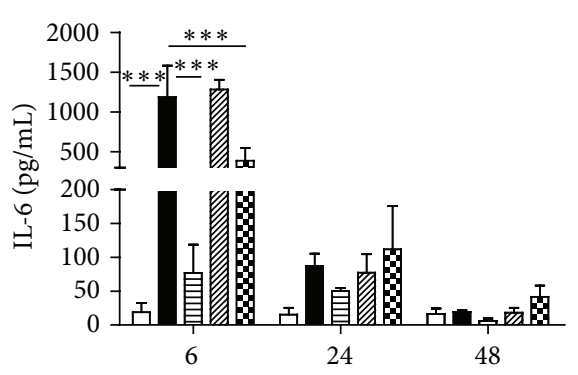

(h)
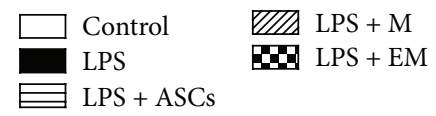

(D)

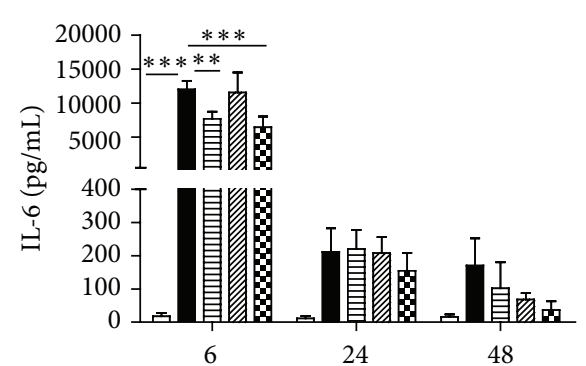

(h)

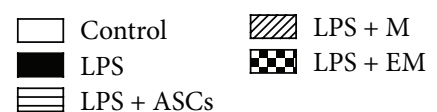

(B)

(a)

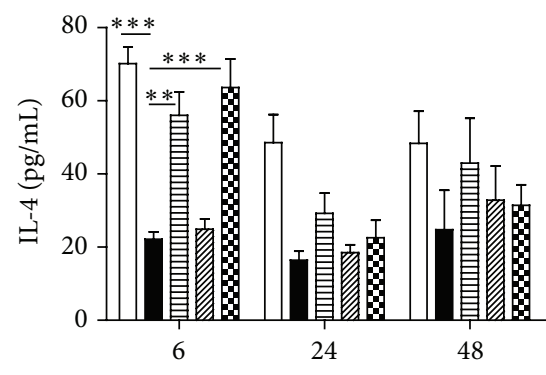

(h)
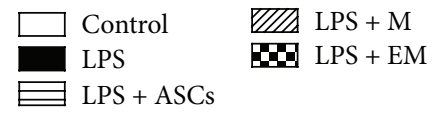

(E)

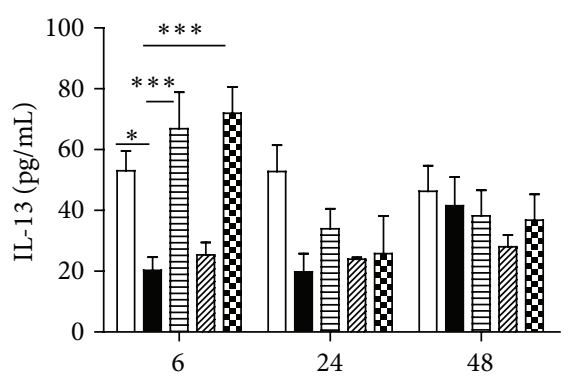

(h)

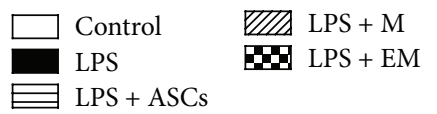

(G)

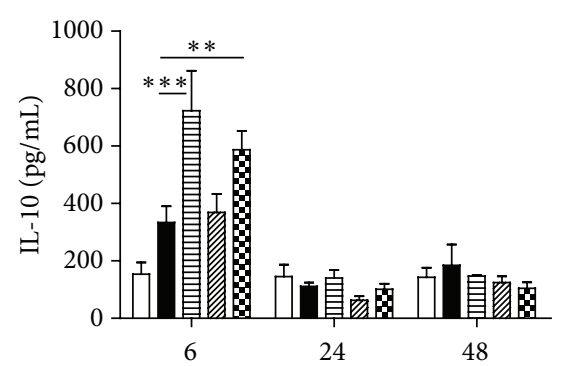

(h)

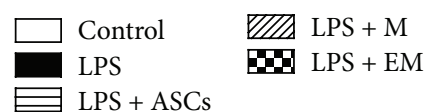

(C)

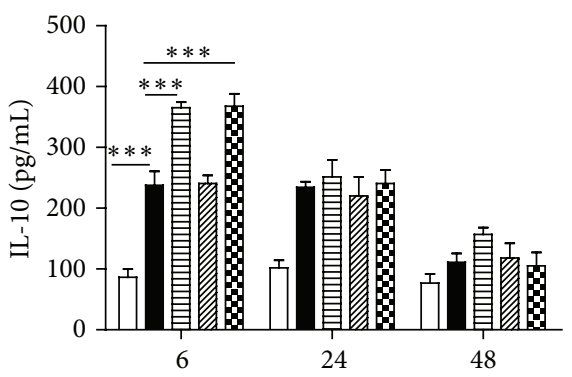

(h)
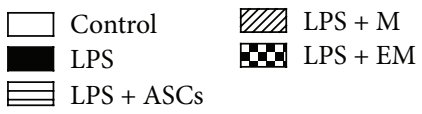

(F)

(b)

FIGURE 3: Infusion of educated macrophages (EM) alters the systemic inflammatory response to LPS. In each group, blood and BAL samples were collected at 6,24 , and $48 \mathrm{~h}$ after LPS or PBS treatment. Cytokine concentrations were determined in a Luminex system. (a) Cytokine levels in serum (IFN- $\gamma$, IL-6, and IL-10). (b) Cytokine levels in BAL (IL-4, IL-6, IL-10, and IL-13). M = macrophages. All data are expressed as mean \pm SEM; $n=4$ per group. ${ }^{*} p<0.05 ;{ }^{* *} p<0.01$; and ${ }^{* * *} p<0.001$.

effects of ASCs on macrophages were blocked by IL-10/IL-10 receptor antibody and mimicked by IL-10.

The direct therapeutic effect of educated macrophages has been documented in two prior studies. In a mouse model of acute kidney injury, animals treated with M0 and M1 macrophages suffered a more severe histological and functional injury, while animals infused with educated macrophages showed mild manifestation [18]. In another report, macrophages were educated via culture with conditioned medium from ASCs [17]. Systemic infusion of the 

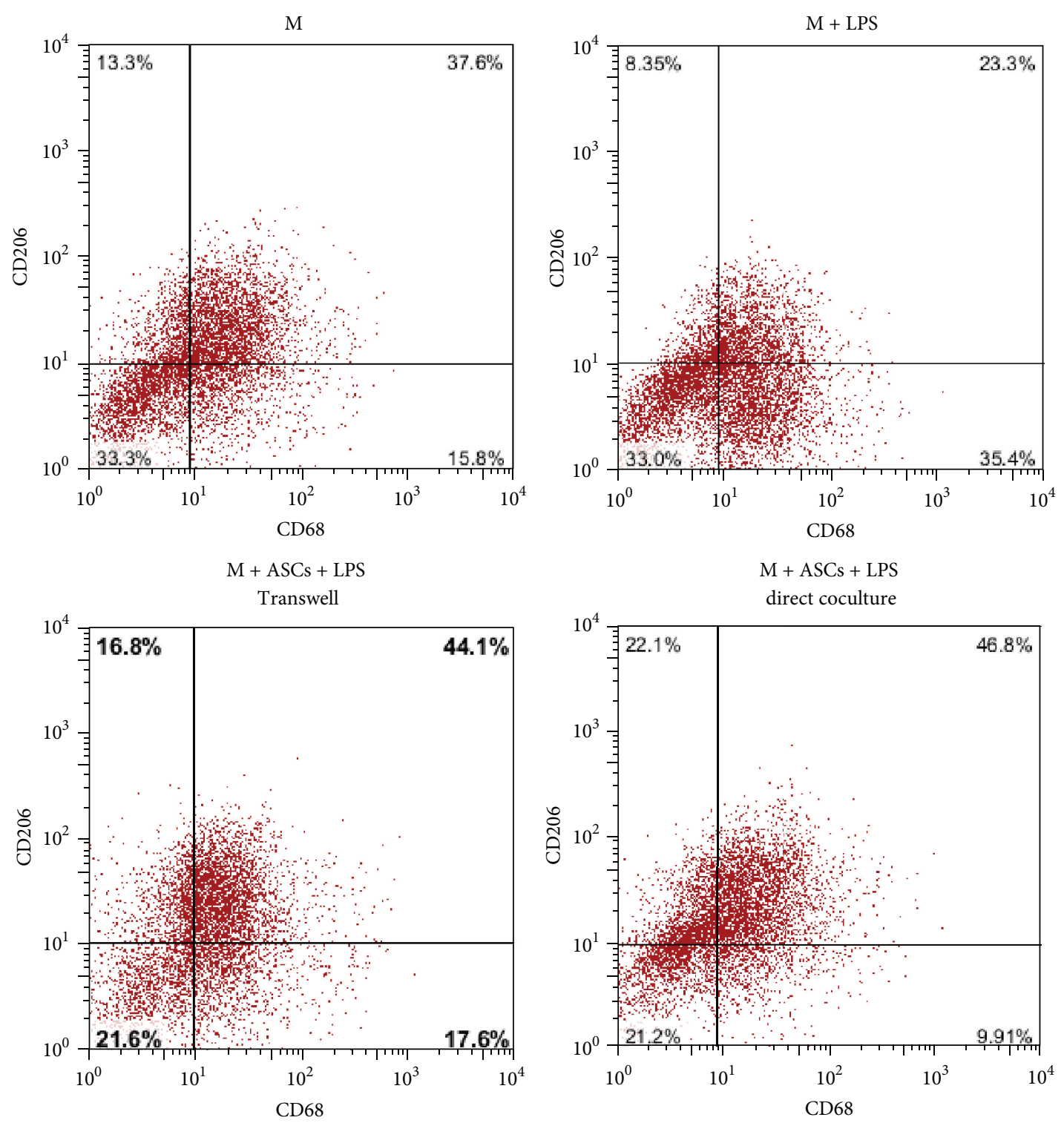

(a)

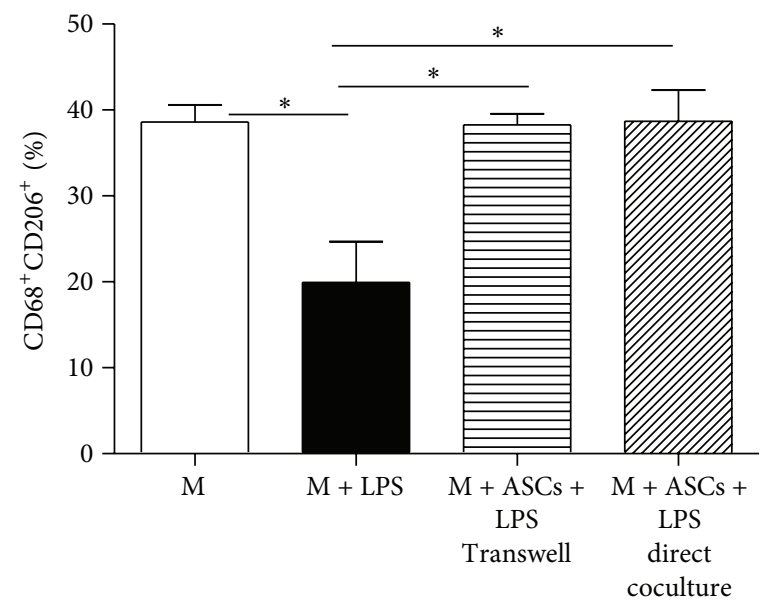

(b)

FIGURE 4: ASCs increase the expression of $\mathrm{CD} 206^{+} \mathrm{CD}^{+} 8^{+}$macrophages independent of direct cell-to-cell contact. (a) Macrophages (M) (2 $\times 10^{6}$ cells/well) were cultured alone or cocultured with ASCs for $48 \mathrm{~h}$ via Transwell or direct culture $(\mathrm{M}:$ ASCs ratio $=4: 1)$. Cells were then washed and incubated with fresh culture medium for $24 \mathrm{~h}$ with or without LPS $(10 \mathrm{ng} / \mathrm{mL})$. The expression of CD206 $6^{+} \mathrm{CD} 68^{+} \mathrm{M}$ was analyzed via flow cytometry. (b) Percentage of $\mathrm{CD} 206^{+} \mathrm{CD}^{+} 8^{+}$macrophages. All data are expressed as mean \pm SEM; $n=4$ per group. ${ }^{*} p<0.05$. 


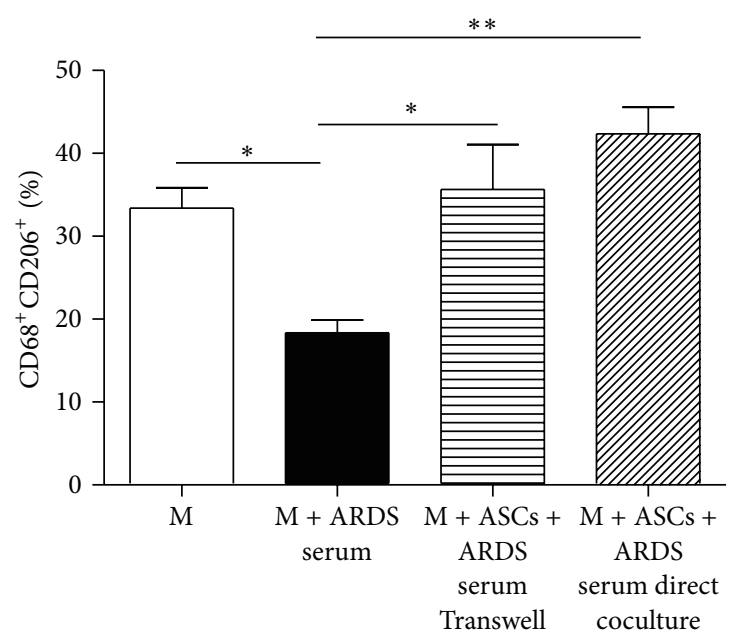

(a)

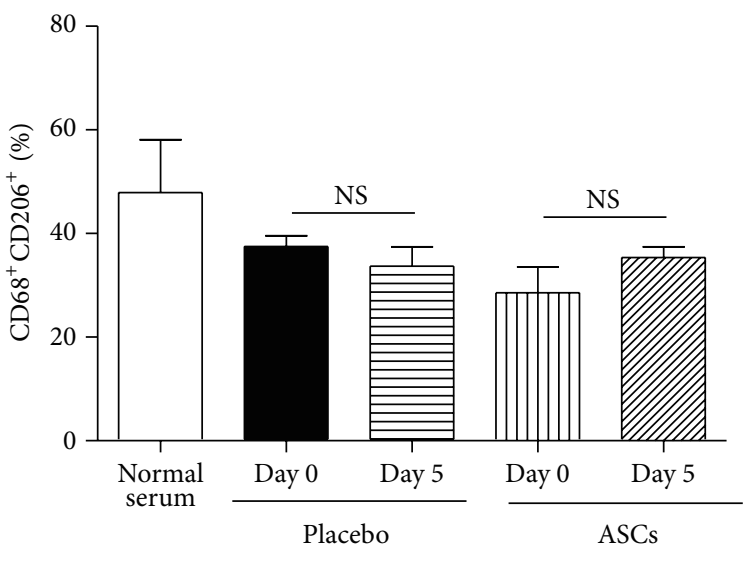

(b)

FIgURE 5: ASCs increase the levels of $\mathrm{CD} 68^{+} \mathrm{CD} 206^{+}$macrophages treated with ARDS serum. (a) Peritoneal macrophages $(\mathrm{M})\left(2 \times 10^{6}\right.$ cells/well) were cultured alone, cocultured directly with ASCs, or cocultured with ASCs via Transwell (M:ASCs ratio = 4:1) for 48 h. Cells were then washed and incubated for $24 \mathrm{~h}$ with fresh culture medium plus $8 \%$ FBS (M group) or $8 \%$ pooled serum from ARDS patients collected at the peak of their clinical symptoms. $n=4$ per group. (b) Serum samples for culture medium (8\%) were from healthy subjects or ARDS patients treated with placebo or ASCs in a previous clinical trial. $n=3$ per group. The expression of CD206 ${ }^{+} \mathrm{CD} 68^{+}$cells was analyzed via flow cytometry. NS = nonsignificant. All data are expressed as mean \pm SEM. ${ }^{*} p<0.05$ and ${ }^{* *} p<0.01$.

educated macrophages inhibited colitis in mice and reduced mortality as well as weight loss while lowering the colonic and systemic levels of inflammatory cytokines. Importantly, therapeutic injection of the macrophages in established chronic colitis alleviated disease progression and avoided recurrence. Moreover, the macrophages protected cecal ligation and puncture-induced sepsis [17].

The concept of educated macrophages was first created by Kim and Hematti. They found that macrophages adopted the phenotypes for M2 after 3 days of coculture with BM-ASCs [15]. González et al. cocultured colitis-derived macrophages with ASCs and found a decrease in the proinflammatory cytokines TNF- $\alpha$ and IL-12 along with an elevation in the anti-inflammatory IL-10. Furthermore, the effects of ASCs on cytokines were reversed by PGE2 blockage [24]. Another group found that BM-MSCs markedly affect the function of macrophages. Educated macrophages had a reduction of the inflammatory cytokines TNF- $\alpha$, IL-6, IL-12p70, and IFN- $\gamma$ and an increase of IL-10 and IL-12p40 when stimulated with LPS [25]. When cocultured with gingival ASCs, Zhang et al. discovered that macrophages acquired an anti-inflammatory M2 phenotype characterized by an increased expression of CD206 and IL-10 as well as a suppressed production of tumor TNF- $\alpha$. In vivo, gingival ASCs homed to the wound site, promoted M2 polarization, and significantly enhanced wound repair. The gingival ASCs-induced suppression of TNF- $\alpha$ secretion by macrophages appears to correlate with impaired activation of $\mathrm{NF} \kappa \mathrm{B}$ p50 [26].

In the present study, we determined that ASCs were able to elevate expression of M2 macrophages and modified cytokine expression without direct cell-to-cell contact. The data suggest that ASCs modulate the function of macrophages via soluble factors or newly defined extracellular vesicles [12]. Our findings were supported by reports from other studies. In an animal model of postspinal cord injury, encapsulated human ASCs were able to modulate the function of inflammatory macrophages and promoted the alternative M2 macrophage phenotype [27]. In vitro, this was evidenced by a reduction in macrophage iNOS expression with a concomitant increase in CD206 [27]. In another study, macrophages cultured with ASCs-conditioned medium showed a M2 phenotype which is characterized by high arginase activity, increased production of IL-10 upon restimulation, and potent immunosuppressive activity on $\mathrm{T}$ cells and macrophages [17]. Although our data showed that both ASCs and educated macrophages were able to reduce LPS-induced systemic response, ASCs might have a better regulatory effect as documented in data from neutrophil infiltration (Figure 1) and cytokine levels (Figure 3). In addition to educated macrophages, ASCs may be able to act via other mechanisms. For example, a number of studies have shown the capacity of mesenchymal stem cells to promote the generation of regulatory T cells (Treg) by activating the Notch 1 signaling pathway [28] or through production of HLA-G5 [29]. Treg itself possesses the ability to ameliorate the LPS response [30].

By depletion of circulating monocytes using clodronate, Németh et al. have demonstrated that BM-MSCs attenuate sepsis via prostaglandin E2-dependent reprogramming of host macrophages to increase their IL-10 production. They propose that MSCs are activated by LPS or TNF- $\alpha$. Then, MSCs reprogram macrophages by releasing prostaglandin E2 that acts on the macrophages through the prostaglandin receptors. Next, activated macrophages produce anti-inflammatory IL-10 which reduce inflammation [10]. M2 macrophage activation has been reported as one of the mechanisms of BM-MSCs in alleviating lung injury by Ionescu et al. [31]. MSCs-derived conditioned medium 

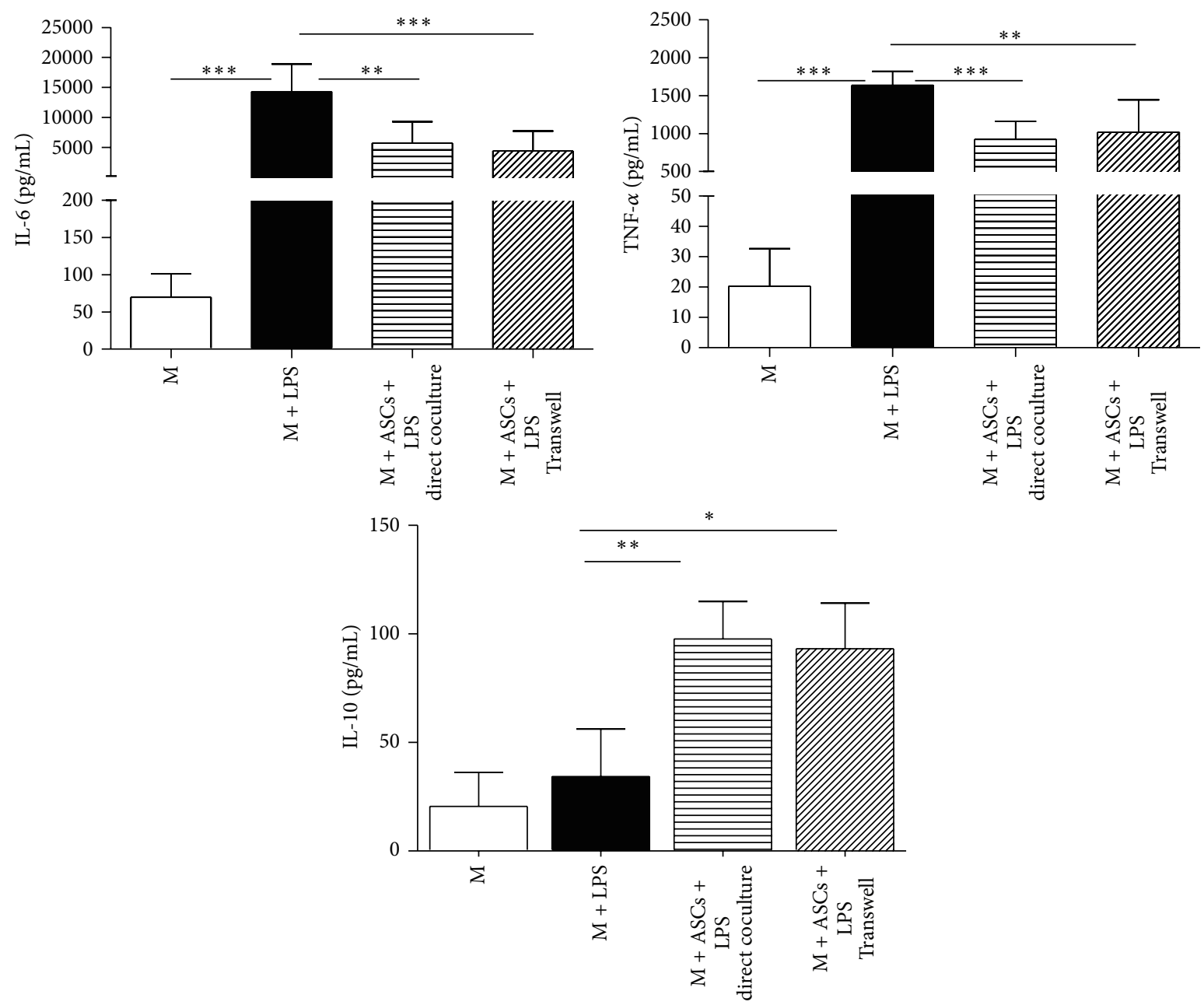

(a)
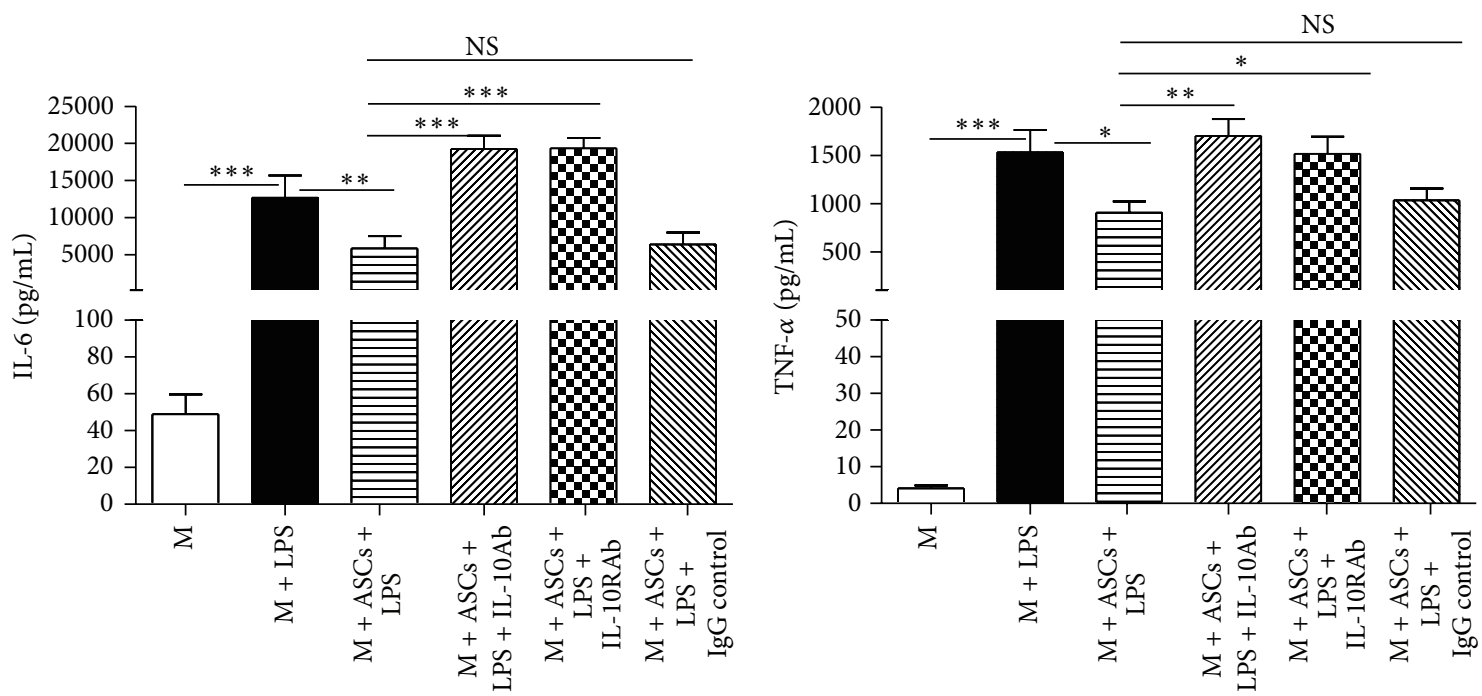

(b)

FIGURE 6: ASCs and IL-10 regulate the profile of cytokines produced by macrophages (M). (a) M ( $2 \times 10^{6}$ cells/well) were cultured alone, cocultured directly with ASCs, or cocultured with ASCs via Transwell (M:ASCs ratio $=4: 1)$ for $48 \mathrm{~h}$. Cells were then washed and incubated for $24 \mathrm{~h}$ with fresh culture medium with or without LPS $(10 \mathrm{ng} / \mathrm{mL}$ ). (b) Neutralizing IL-10 antibody (IL-10Ab, $10 \mathrm{ng} / \mathrm{mL}$ ), IL-10 receptor antibody (IL-10RAb, $10 \mathrm{ng} / \mathrm{mL}$ ), or IgG control $(10 \mathrm{ng} / \mathrm{mL})$ was added to the medium at the beginning of the Transwell cocultures of ASCs + M. Then, cells were treated with or without LPS $(10 \mu \mathrm{g} / \mathrm{mL})$ for another $24 \mathrm{~h}$. Culture supernatants were harvested for cytokine assay via Luminex. All data are expressed as mean $\pm \mathrm{SEM} ; n=4$ per group. ${ }^{*} p<0.05 ;{ }^{* *} p<0.01$; and ${ }^{* * *} p<0.001$. 

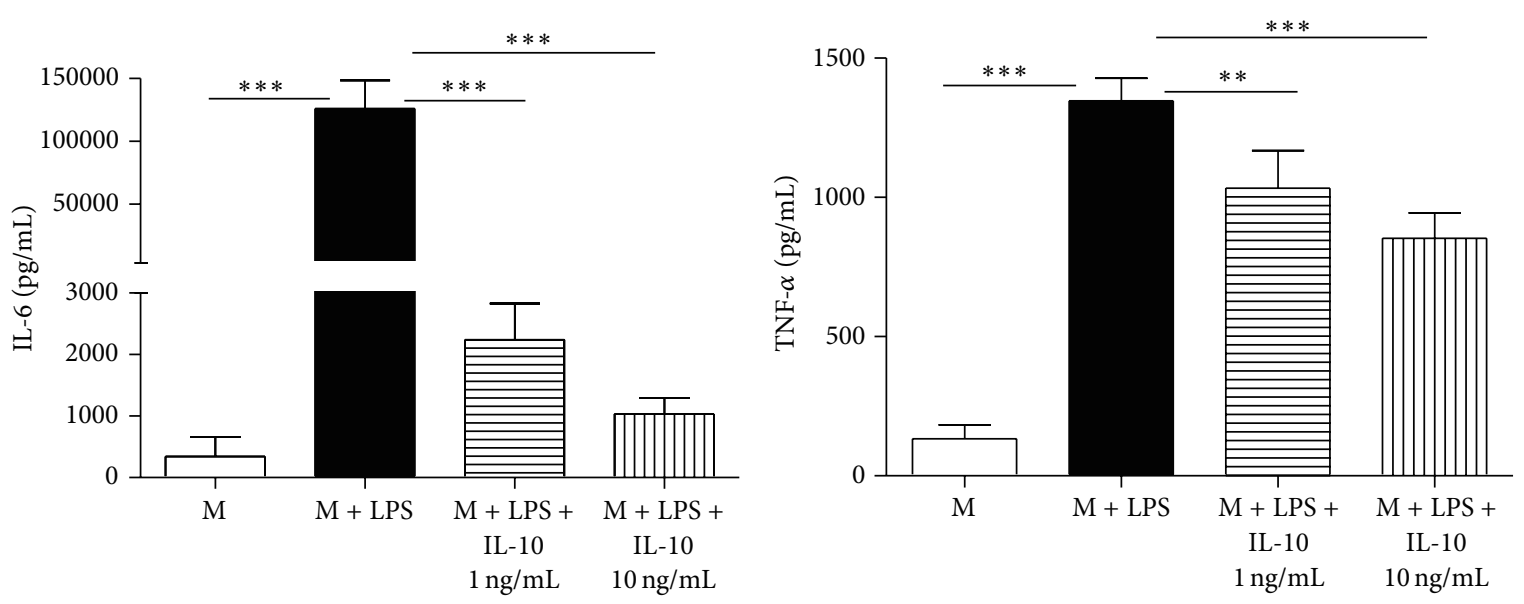

(a)

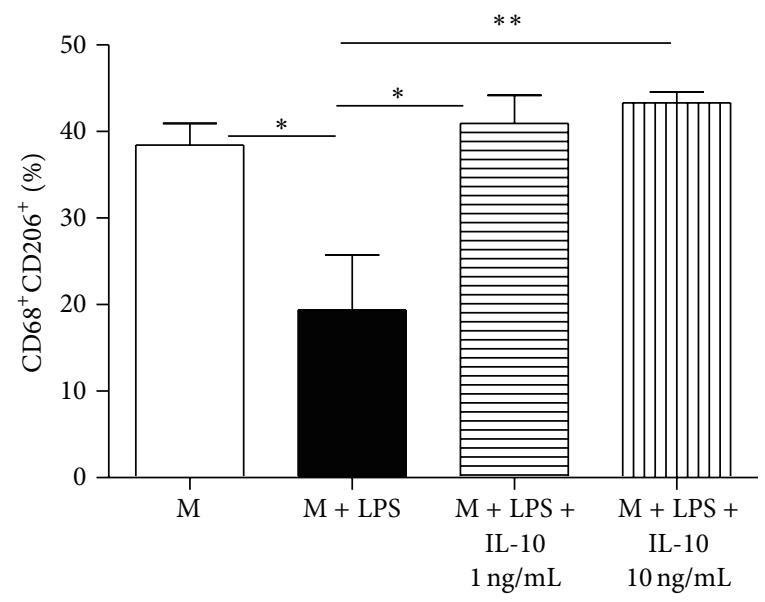

(b)

FIGURE 7: IL-10 mimics the effects of educated macrophages. (a) $\mathrm{M}\left(2 \times 10^{6}\right.$ cells/well) were pretreated with or without IL-10 (1 ng/mL and $10 \mathrm{ng} / \mathrm{mL})$ for 6 hours and then incubated with LPS $(10 \mathrm{ng} / \mathrm{mL})$ for another 24 hours. Culture supernatants were harvested for cytokine assay via Luminex. (b) Percentage of $\mathrm{CD} 8^{+} \mathrm{CD} 206^{+}$macrophages after IL-10 and LPS treatment was determined via flow cytometry. All data are expressed as mean \pm SEM; $n=4$ per group. ${ }^{*} p<0.05 ;^{* *} p<0.01$; and ${ }^{* * *} p<0.001$.

(MSCs-CdM) promoted the resolution of LPS-induced lung injury by increasing a wound healing/anti-inflammatory M2 macrophage phenotype. MSCs-CdM increased arginase1 activity and Yml expression in LPS-exposed alveolar macrophages. In vivo, alveolar macrophages from LPS-MSCs and LPS-MSCs-CdM lungs had enhanced expression of Ym1 and decreased expression of inducible nitric oxide synthase compared with untreated LPS mice. This suggests that MSCs$\mathrm{CdM}$ promotes the alternative macrophage activation to an M2 "healer" phenotype. Furthermore, recombinant insulinlike growth factor I (IGF-I) partially reproduced the lung protective effect of MSCs-CdM [31]. By injecting ASCseducated macrophages into mice treated with LPS, the present study showed that educated macrophages alone can directly ameliorate systemic inflammation. This finding may provide new insights into the mechanisms of MSCs and systemic inflammation.

The use of allogeneic MSCs holds great promise as a treatment for ARDS, sepsis, and systemic inflammation due to time limitations and the difficulties involved with harvesting autologous BM-MSCs or ASCs in these critical ill patients. Allogeneic BM-MSCs and ASCs have been efficiently used without major histocompatibility complex matching in clinical studies such as graft-versus-host disease [32], myocardial infarction [33], and inflammatory bowel diseases [34]. In a study of two ARDS patients administered with a dose of $2 \times 10^{6}$ MSCs cells per kilogram, both patients improved with resolution of respiratory, hemodynamic, and multiorgan failure [35]. Our group previously conducted a pilot clinical study in the treatment of ARDS with $1 \times 10^{6}$ ASCs cells per kilogram. The results showed that there were no short-term toxicities or serious adverse events related to ASCs administration. In the ASCs group, serum SP-D levels at day 5 were significantly lower than those at day $0(p=0.027)$ while the changes in IL-8 levels were not significant. The IL- 6 levels at day 5 showed a trend towards lower levels as compared with day 0 , but this trend was not statistically significant $(p=0.06)$. However, length of hospital stay, ventilator-free days, and ICU-free days at day 28 after treatment were similar between ASCs and control 
group [20]. In the present study, when macrophages were treated with serum from the trial, there was no difference in M2 macrophage levels before and after ASCs treatment. This finding further indicates that our trial protocol is not optimal. A higher or more frequent dose may be warranted.

Based on the findings of the present study, educated macrophages can be obtained by $48 \mathrm{~h}$ of coculture ASCs with macrophages, which can readily be generated from peripheral blood monocytes. Therefore, educated macrophages may serve as an attractive and alternative treatment option for ARDS, sepsis, and systemic inflammation. In comparison with BM-MSCs or ASCs, treatments based on educated macrophages are limited to their use only in an autologous manner. This suggests an obvious disadvantage versus treatments with allogeneic BM-MSCs or ASCs. However, the long-term safety of BM-MSCs and ASCs is unknown due to concern for carcinogenesis [36]. Moreover, autologous macrophages have been successfully used for promoting transplant tolerance [37] and the treatment of chronic wounds [38] in clinical trials.

Our results demonstrate that administration of ASCseducated macrophages reduce systemic inflammation induced by LPS. The data also reveal that ASCs favor the expression of M2 phenotype and anti-inflammatory cytokines independent of direct cell-to-cell contact. Furthermore, educated macrophages may function via IL-10 pathways. Further studies are warranted to delineate the mechanisms of educated macrophages on systemic inflammation.

\section{Competing Interests}

The authors declare that they have no competing interests.

\section{Acknowledgments}

This work was supported by the Zhejiang Health Bureau Grant 2015KYB194 to Yaoqin $\mathrm{Hu}$; the National Natural Science Foundation of China (81272139) and the Zhejiang Provincial Program for the Cultivation of High-level Innovative Health Talents (2016-6) to Qiang Shu; and the National Natural Science Foundation of China (81270068 and 81570071), Zhejiang Health Bureau Cultivation Plan (2014PYA020), and Zhejiang Science and Technology Program (2015C33256) to Jianguo Xu.

\section{References}

[1] J. Xu, C. R. Woods, A. L. Mora et al., "Prevention of endotoxininduced systemic response by bone marrow-derived mesenchymal stem cells in mice," American Journal of Physiology - Lung Cellular and Molecular Physiology, vol. 293, no. 1, pp. L131-L141, 2007.

[2] M. Rojas, J. Xu, C. R. Woods et al., "Bone marrow-derived mesenchymal stem cells in repair of the injured lung," American Journal of Respiratory Cell and Molecular Biology, vol. 33, no. 2, pp. 145-152, 2005.

[3] S. H. J. Mei, S. D. McCarter, Y. Deng, C. H. Parker, W. C. Liles, and D. J. Stewart, "Prevention of LPS-induced acute lung injury in mice by mesenchymal stem cells overexpressing angiopoietin," PLoS Medicine, vol. 4, no. 9, article e269, 2007.

[4] L. A. Ortiz, F. Gambelli, C. McBride et al., "Mesenchymal stem cell engraftment in lung is enhanced in response to bleomycin exposure and ameliorates its fibrotic effects," Proceedings of the National Academy of Sciences of the United States of America, vol. 100, no. 14, pp. 8407-8411, 2003.

[5] N. Gupta, X. Su, B. Popov, W. L. Jae, V. Serikov, and M. A. Matthay, "Intrapulmonary delivery of bone marrow-derived mesenchymal stem cells improves survival and attenuates endotoxin-induced acute lung injury in mice," Journal of Immunology, vol. 179, no. 3, pp. 1855-1863, 2007.

[6] C.-K. Sun, C.-H. Yen, Y.-C. Lin et al., "Autologous transplantation of adipose-derived mesenchymal stem cells markedly reduced acute ischemia-reperfusion lung injury in a rodent model," Journal of Translational Medicine, vol. 9, article 118, 2011.

[7] I. Martínez-González, O. Roca, J. R. Masclans et al., "Human mesenchymal stem cells overexpressing the IL-33 antagonist soluble IL-1 receptor-like-1 attenuate endotoxin-induced acute lung injury," American Journal of Respiratory Cell and Molecular Biology, vol. 49, no. 4, pp. 552-562, 2013.

[8] M.-H. Chien, M.-Y. Bien, C.-C. Ku et al., "Systemic human orbital fat-derived stem/stromal cell transplantation ameliorates acute inflammation in lipopolysaccharide-induced acute lung injury," Critical Care Medicine, vol. 40, no. 4, pp. 1245-1253, 2012.

[9] J. Chen, C. Li, X. Gao et al., "Keratinocyte growth factor gene delivery via mesenchymal stem cells protects against lipopolysaccharide-induced acute lung injury in mice," PLoS ONE, vol. 8, no. 12, Article ID e83303, 2013.

[10] K. Németh, A. Leelahavanichkul, P. S. T. Yuen et al., "Bone marrow stromal cells attenuate sepsis via prostaglandin $\mathrm{E}(2)$ dependent reprogramming of host macrophages to increase their interleukin-10 production," Nature Medicine, vol. 15, no. 1, pp. 42-49, 2009.

[11] J. W. Lee, A. Krasnodembskaya, D. H. McKenna, Y. Song, J. Abbott, and M. A. Matthay, "Therapeutic effects of human mesenchymal stem cells in ex vivo human lungs injured with live bacteria," American Journal of Respiratory and Critical Care Medicine, vol. 187, no. 7, pp. 751-760, 2013.

[12] D. G. Phinney, M. Di Giuseppe, J. Njah et al., "Mesenchymal stem cells use extracellular vesicles to outsource mitophagy and shuttle microRNAs," Nature Communications, vol. 6, article 8472, 2015.

[13] S. Herold, K. Mayer, and J. Lohmeyer, "Acute lung injury: how macrophages orchestrate resolution of inflammation and tissue repair," Frontiers in Immunology, vol. 2, article 65, 2011.

[14] P. Salgame, G. S. Yap, and W. C. Gause, "Effect of helminthinduced immunity on infections with microbial pathogens," Nature Immunology, vol. 14, no. 11, pp. 1118-1126, 2013.

[15] J. Kim and P. Hematti, "Mesenchymal stem cell-educated macrophages: a novel type of alternatively activated macrophages," Experimental Hematology, vol. 37, no. 12, pp. 1445-1453, 2009.

[16] E. Eggenhofer and M. J. Hoogduijn, "Mesenchymal stem celleducated macrophages," Transplantation Research, vol. 1, article 12, 2012.

[17] P. Anderson, L. Souza-Moreira, M. Morell et al., "Adiposederived mesenchymal stromal cells induce immunomodulatory macrophages which protect from experimental colitis and sepsis," Gut, vol. 62, no. 8, pp. 1131-1141, 2013.

[18] Y. Geng, L. Zhang, B. Fu et al., "Mesenchymal stem cells ameliorate rhabdomyolysis-induced acute kidney injury via the 
activation of M2 macrophages," Stem Cell Research \& Therapy, vol. 5, article 80, 2014.

[19] V. M. Ranieri, G. D. Rubenfeld, B. T. Thompson et al., "Acute respiratory distress syndrome: the Berlin definition," The Journal of the American Medical Association, vol. 307, no. 23, pp. 25262533, 2012.

[20] G. Zheng, L. Huang, H. Tong et al., "Treatment of acute respiratory distress syndrome with allogeneic adipose-derived mesenchymal stem cells: a randomized, placebo-controlled pilot study," Respiratory Research, vol. 15, article 39, 2014.

[21] M. B. Everhart, W. Han, T. P. Sherrill et al., "Duration and intensity of NF- $\kappa$ B activity determine the severity of endotoxininduced acute lung injury," Journal of Immunology, vol. 176, no. 8, pp. 4995-5005, 2006.

[22] S. Zhang, S. D. Danchuk, K. M. Imhof et al., "Comparison of the therapeutic effects of human and mouse adipose-derived stem cells in a murine model of lipopolysaccharide-induced acute lung injury," Stem Cell Research \& Therapy, vol. 4, article 13, 2013.

[23] Y. Zhang, W. Sime, M. Juhas, and A. Sjölander, "Crosstalk between colon cancer cells and macrophages via inflammatory mediators and CD47 promotes tumour cell migration," European Journal of Cancer, vol. 49, no. 15, pp. 3320-3334, 2013.

[24] M. A. González, E. Gonzalez-Rey, L. Rico, D. Büscher, and M. Delgado, "Adipose-derived mesenchymal stem cells alleviate experimental colitis by inhibiting inflammatory and autoimmune responses," Gastroenterology, vol. 136, no. 3, pp. 978-989, 2009.

[25] J. Maggini, G. Mirkin, I. Bognanni et al., "Mouse bone marrowderived mesenchymal stromal cells turn activated macrophages into a regulatory-like profile," PLOS ONE, vol. 5, no. 2, Article ID e9252, 2010.

[26] Q.-Z. Zhang, W.-R. Su, S.-H. Shi et al., "Human gingiva-derived mesenchymal stem cells elicit polarization of M2 macrophages and enhance cutaneous wound healing," Stem Cells, vol. 28, no. 10, pp. 1856-1868, 2010.

[27] J. Barminko, J. H. Kim, S. Otsuka et al., "Encapsulated mesenchymal stromal cells for in vivo transplantation," Biotechnology and Bioengineering, vol. 108, no. 11, pp. 2747-2758, 2011.

[28] B. Del Papa, P. Sportoletti, D. Cecchini et al., "Notch1 modulates mesenchymal stem cells mediated regulatory T-cell induction," European Journal of Immunology, vol. 43, no. 1, pp. 182-187, 2013.

[29] Z. Selmani, A. Naji, I. Zidi et al., "Human leukocyte antigenG5 secretion by human mesenchymal stem cells is required to suppress T lymphocyte and natural killer function and to induce $\mathrm{CD}^{+}{ }^{+} \mathrm{CD} 25$ highFOXP3+ regulatory T cells," Stem Cells, vol. 26, no. 1, pp. 212-222, 2008.

[30] E. B. Okeke, I. Okwor, Z. Mou, P. Jia, and J. E. Uzonna, " $\mathrm{CD} 4{ }^{+} \mathrm{CD} 25^{+}$regulatory $\mathrm{T}$ cells attenuate lipopolysaccharideinduced systemic inflammatory responses and promotes survival in murine Escherichia coli infection," Shock, vol. 40, no. 1, pp. 65-73, 2013.

[31] L. Ionescu, R. N. Byrne, T. van Haaften et al., "Stem cell conditioned medium improves acute lung injury in mice: in vivo evidence for stem cell paracrine action," American Journal of Physiology_Lung Cellular and Molecular Physiology, vol. 303, no. 11, pp. L967-L977, 2012.

[32] K. Le Blanc, F. Frassoni, L. Ball et al., "Mesenchymal stem cells for treatment of steroid-resistant, severe, acute graft-versushost disease: a phase II study,' The Lancet, vol. 371, no. 9624, pp. 1579-1586, 2008.

[33] J. M. Hare, J. H. Traverse, T. D. Henry et al., "A randomized, double-blind, placebo-controlled, dose-escalation study of intravenous adult human mesenchymal stem cells (prochymal) after acute myocardial infarction," Journal of the American College of Cardiology, vol. 54, no. 24, pp. 2277-2286, 2009.

[34] F. de la Portilla, F. Alba, D. García-Olmo, J. M. Herrerías, F. X. González, and A. Galindo, "Expanded allogeneic adiposederived stem cells (eASCs) for the treatment of complex perianal fistula in Crohn's disease: results from a multicenter phase I/IIa clinical trial," International Journal of Colorectal Disease, vol. 28, no. 3, pp. 313-323, 2013.

[35] O. E. Simonson, D. Mougiakakos, N. Heldring et al., "In vivo effects of mesenchymal stromal cells in two patients with severe acute respiratory distress syndrome," Stem Cells Translational Medicine, vol. 4, no. 10, pp. 1199-1213, 2015.

[36] M. Miura, Y. Miura, H. M. Padilla-Nash et al., "Accumulated chromosomal instability in murine bone marrow mesenchymal stem cells leads to malignant transformation," Stem Cells, vol. 24, no. 4, pp. 1095-1103, 2006.

[37] J. A. Hutchinson, P. Riquelme, B. Sawitzki et al., "Cutting edge: immunological consequences and trafficking of human regulatory macrophages administered to renal transplant recipients," Journal of Immunology, vol. 187, no. 5, pp. 2072-2078, 2011.

[38] A. Zuloff-Shani, A. Adunsky, A. Even-Zahav et al., "Hard to heal pressure ulcers (stage III-IV): efficacy of injected activated macrophage suspension (AMS) as compared with standard of care (SOC) treatment controlled trial," Archives of Gerontology and Geriatrics, vol. 51, no. 3, pp. 268-272, 2010. 


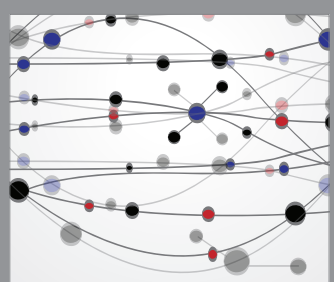

The Scientific World Journal
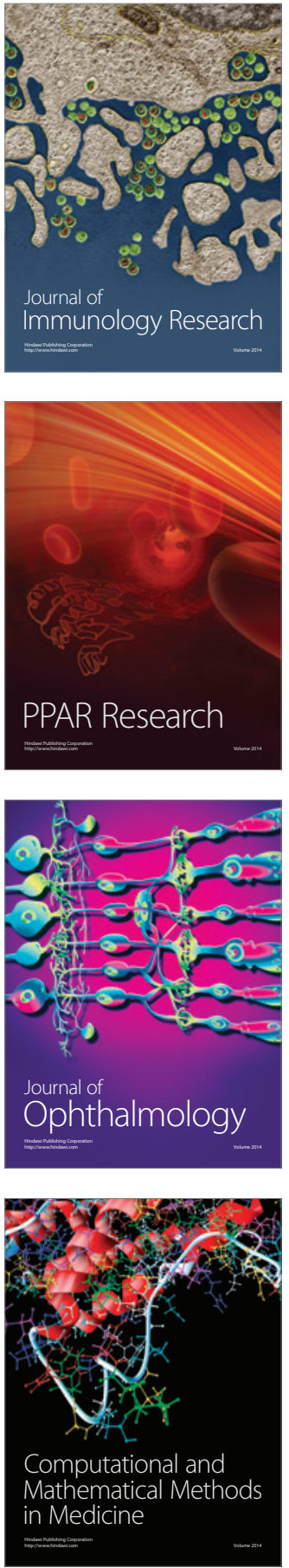

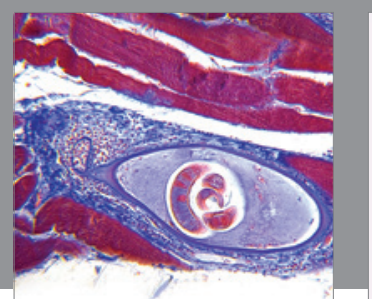

Gastroenterology Research and Practice

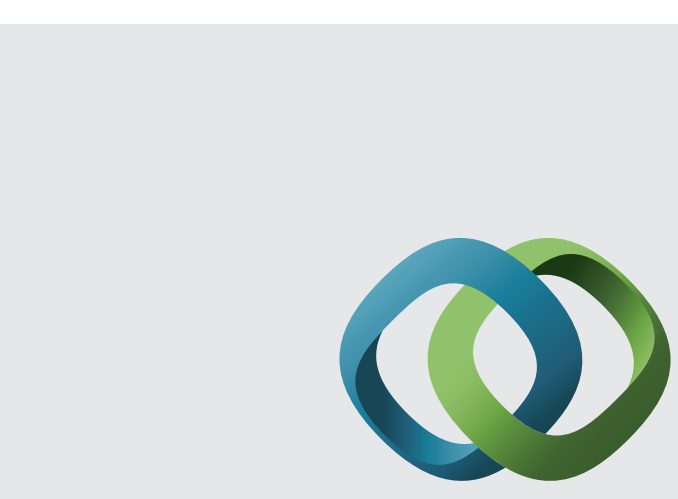

\section{Hindawi}

Submit your manuscripts at

http://www.hindawi.com
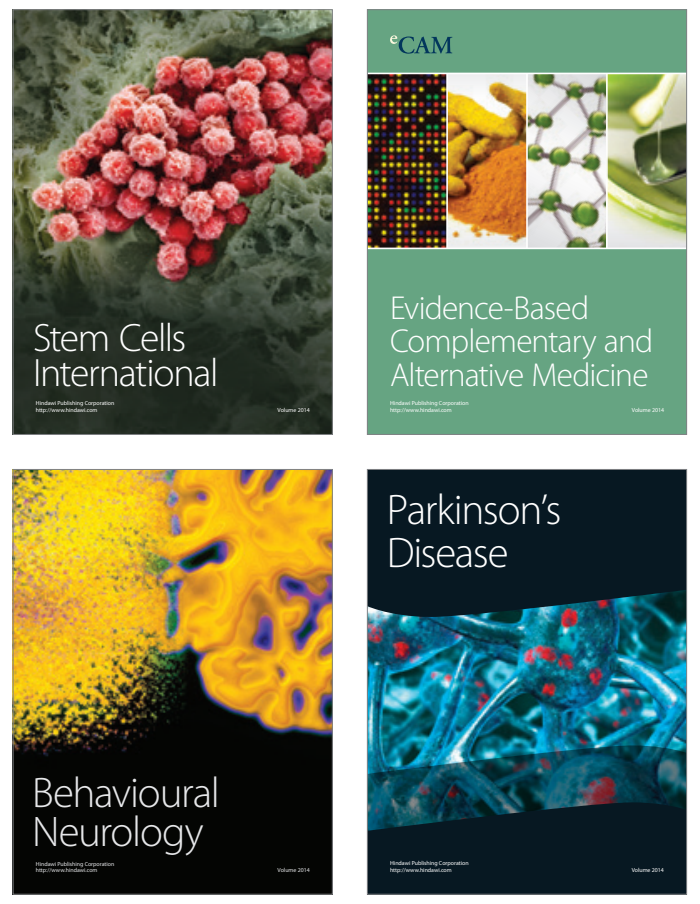
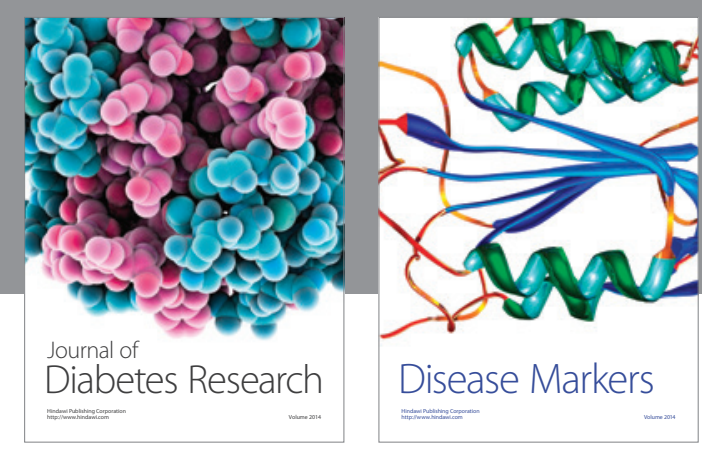

Disease Markers
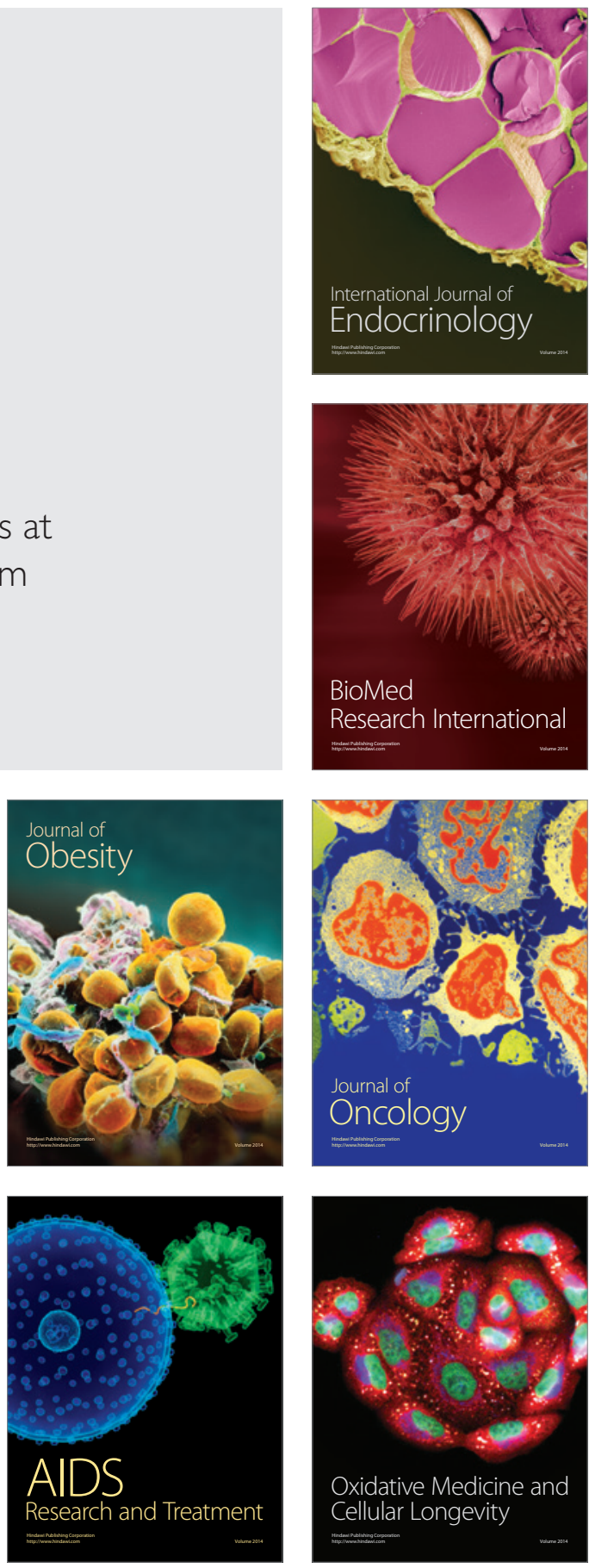